\title{
BMJ Open Racial/ethnic heterogeneity in associations of blood pressure and incident cardiovascular disease by functional status in a prospective cohort: the Multi- Ethnic Study of Atherosclerosis
}

\author{
Paulina Kaiser, ${ }^{1}$ Carmen A. Peralta, ${ }^{2}$ Richard Kronmal, ${ }^{3}$ Michael G. Shlipak, ${ }^{2,4}$ \\ Bruce M Psaty, ${ }^{5}$ Michelle C Odden ${ }^{1}$
}

To cite: Kaiser P, Peralta CA., Kronmal $\mathrm{R}$, et al. Racial/ ethnic heterogeneity in associations of blood pressure and incident cardiovascular disease by functional status in a prospective cohort: the Multi-Ethnic Study of Atherosclerosis. BMJ Open 2018;8:e017746. doi:10.1136/ bmjopen-2017-017746

- Prepublication history and additional material for this paper are available online. To view these files, please visit the journal online (http://dx.doi org/10.1136/bmjopen-2017017746).

Received 24 May 2017 Revised 21 December 2017 Accepted 12 January 2018

Check for updates

For numbered affiliations see end of article.

Correspondence to

Dr Paulina Kaiser;

paulina.kaiser@oregonstate.edu

\section{ABSTRACT}

Objectives Research has demonstrated that the association between high blood pressure and outcomes is attenuated among older adults with functional limitations, compared with healthier elders. However, it is not known whether these patterns vary by racial/ethnic group. We evaluated race/ethnicity-specific patterns of effect modification in the association between blood pressure and incident cardiovascular disease (CVD) by functional status.

Setting We used data from the Multi-Ethnic Study of Atherosclerosis (2002-2004, with an average of 8.8 years of follow-up for incident CVD). We assessed effect modification of systolic blood pressure and cardiovascular outcomes by self-reported physical limitations and by age.

Participants The study included 6117 participants (aged 46 to $87 ; 40 \%$ white, $27 \%$ black, $22 \%$ Hispanic and $12 \%$ Chinese) who did not have CVD at the second study examination (when self-reported physical limitations were assessed).

Outcome measures Incident CVD was defined as an incident myocardial infarction, coronary revascularisation, resuscitated cardiac arrest, angina, stroke (fatal or nonfatal) or death from CVD.

Results We observed weaker associations between systolic blood pressure (SBP) and CVD among white adults with physical limitations (incident rate ratio (IRR) per $10 \mathrm{~mm} \mathrm{Hg}$ higher SBP: 1.09 (95\% Cl 0.99 to 1.20)) than those without physical limitations (IRR 1.29 (1.19, 1.40); $P$ value for interaction $<0.01)$. We found a similar pattern among black adults. Poor precision among the estimates for Hispanic or Chinese participants limited the findings in these groups. The attenuated associations were consistent across both multiplicative and additive scales, though physical limitations showed clearer patterns than age on an additive scale.

Conclusion Attenuated associations between high blood pressure and incident CVD were observed for blacks and whites with poor function, though small sample sizes remain a limitation for identifying differences among Hispanic or Chinese participants. Identifying the characteristics that distinguish those in whom higher SBP is associated with less risk of morbidity or mortality
Strengths and limitations of this study

- The Multi-Ethnic Study of Atherosclerosis is the largest cohort of middle-aged and older adults in the USA from four racial/ethnic groups (white, black, Hispanic and Chinese).

- We evaluated self-reported physical limitations (based on two questions) and chronological age as potential effect modifiers of systolic blood pressure (SBP) and cardiovascular outcomes.

- Small sample size among some racial/ethnic groups limited the precision of our estimates.

- Additional research is needed to investigate heterogeneity in associations between SBP and cardiovascular disease among racial/ethnic minority populations, and to understand the underlying biological mechanisms.

may inform our understanding of the consequences of hypertension among older adults.

\section{INTRODUCTION}

High blood pressure (systolic blood pressure $(\mathrm{SBP}) \geq 140 \mathrm{~mm} \mathrm{Hg}$ or diastolic blood pressure $(\mathrm{DBP}) \geq 90 \mathrm{~mm} \mathrm{Hg}$ or taking antihypertensive medicine) is a major cause of morbidity and mortality among American adults, and is responsible for an estimated US $\$ 48.6$ billion in direct and indirect costs per year. ${ }^{1}$ However, the health risks associated with high blood pressure are not uniform in all adults; some subgroups have been identified in which elevated blood pressure is not associated with increased morbidity or mortality. For example, among older adults with poor functional status, measured by slow gait speed $^{23}$ or by functional limitations, the association between high blood pressure and mortality is attenuated compared with elders with better functional status. ${ }^{4}$ Interestingly, 
in this latter study, the effect modification of blood pressure and mortality by functional status was less apparent in middle-aged participants. ${ }^{4}$ There are limited data on functional status as an effect modifier of blood pressure and cardiovascular outcomes; existing studies have been limited to older adults. ${ }^{56}$ Thus, it is not yet clear how age and functional limitations pattern risks of cardiovascular disease (CVD) associated with elevated SBP.

Additionally, it is plausible that the patterns of blood pressure, functional status and morbidity vary by racial/ ethnic group. Previous research has demonstrated patterns of attenuation in populations of Latinos ${ }^{2}$ and populations of white Americans and African Americans. ${ }^{45}$ Race/ethnicity, as a proxy for biological characteristics as well as lifelong contextual influences, cultural norms and cumulative stressors, ${ }^{7}$ is associated with blood pressure levels and with rates of CVD. For example, a $10 \mathrm{~mm} \mathrm{Hg}$ higher SPB level is associated with a larger increase in stroke risk for blacks than for whites. ${ }^{8}$ Our objective was to explore racial/ethnic heterogeneity in the interaction of blood pressure and functional status on incident CVD.

\section{METHODS}

\section{Study population}

We used data from the Multi-Ethnic Study of Atherosclerosis (MESA) to explore race/ethnicity-specific patterns of effect modification in the association between blood pressure and incident CVD by physical limitations and by age.

MESA includes 6814 adults aged $45-84$ who self-identified as white, black, Hispanic or Chinese from six areas across the USA (New York, New York; Baltimore, Maryland; Forsyth County, North Carolina; Chicago, Illinois; St Paul, Minnesota; and Los Angeles, California). White participants were recruited at all sites; black participants were recruited at all sites except for Minnesota. Hispanic participants came from New York, Minnesota and California, and Chinese participants came from Chicago and California. Participants were excluded if they had a history of heart attack, angina, stroke or transient ischaemic attack, heart failure, resuscitated cardiac arrest or procedures related to CVD. Participants were followed from 2000-2002 until 2010-2012, with a total of five study examinations. Retention was $92.4 \%$ from the first examination to the second examination, and $75.7 \%$ from the fourth examination to the final examination. ${ }^{9}$ The participants provided informed consent. ${ }^{10}$

\section{Exposure: SBP}

Blood pressure was measured at each examination, following a standardised protocol. After $5 \mathrm{~min}$ of seated rest, SBP and DBP were measured three times, at $2 \mathrm{~min}$ intervals, using an automated oscillometric sphygmomanometer. ${ }^{11}$ The average of the second and third measurements was used for analysis. We used SBP measured at the second examination (2002-2004) as the primary exposure of interest because physical limitations were not assessed at the baseline examination.

\section{Effect modifiers}

We used two effect modifiers for this analysis: self-reported physical limitations and age. Physical limitations were assessed at examination 2, in 2002-2004, and measured with two questions based off the same prompt: 'During the past 4 weeks, have you had any of the following problems with your work or other regular daily activities as a result of your physical health?' Participants were considered to have physical limitations if they answered 'yes' to either 'You accomplished less than you would have liked to' or 'You were limited in the kind of work you do or other regular daily activities.'

As a secondary analysis, we used summary scores from a modified version of the 12-Item Short Form Health Survey (SF-12) (version 2) ${ }^{12}$ to measure overall physical health and well-being. The SF-12 survey is a shortened version of the SF-36 scale and has been validated in a variety of settings. ${ }^{13} 14$ The Physical Component Summary score (SF12-P) is a weighted average of questions about general health, limitations in moderate activities or climbing stairs, physical limitations, emotional limitations, pain interfering with work, feeling downhearted and blue and health interfering with social activities. SF12-P scores range from 0 to 100 (higher scores represent better function) and are standardised so that a score of 50 reflects the average of the general US population. ${ }^{15}$ The SF12-P scale was used as a secondary analysis because it includes the physical limitation questions described above, in addition to other covariates that were less related to the underlying construct of functional status.

\section{Outcome: incident CVD}

The outcome of interest was incident CVD, measured through the end of 2012. MESA participants were contacted by phone every 9 to 12 months and asked about interim cardiovascular events. Medical records, death certificates and next-of-kin interviews (for out-of-hospital cardiovascular deaths) were used by a team of two study cardiologists, cardiovascular epidemiologists or neurologists to determine the date of incident CVD. ${ }^{16}$ CVD was defined as an incident myocardial infarction, coronary revascularisation, resuscitated cardiac arrest, angina, stroke (fatal or non-fatal) or CVD death. ${ }^{17}$

\section{Covariates}

All covariates were measured at the second examination (2002-2004). Demographic confounders included age, sex and income. Income was dichotomised for this analysis at earning US $\$ 75000$ per year. Additional covariates included smoking (never, former or current smoker), body mass index (BMI), total cholesterol, diabetes status and medication use. Diabetes status was categorised as normal, impaired fasting glucose or untreated diabetes and treated diabetes based on the 2003 American Diabetes Association fasting criteria algorithm. 
Medications (antihypertensives and statins) were assessed by visual inspection of medication containers by study personnel or by self-report.

\section{Statistical methods}

Participants with missing data on SBP or physical limitations at examination 2, or on incident CVD status, were excluded, as were participants who had developed CVD by examination 2 . We first stratified the study population by race/ethnicity, and summarised blood pressure, functional status and other covariates within each racial/ ethnic group. Then we used Poisson models to estimate the incident rate ratios for incident CVD per $10 \mathrm{~mm} \mathrm{Hg}$ higher SBP, using an offset for the person-time contributed until incident CVD, death or lost to follow-up. A likelihood ratio test provided evidence for a three-way interaction (on a multiplicative scale) between race/ ethnicity, age and SBP $(\mathrm{P}=0.07)$, so we present models stratified on race/ethnicity.

All models were adjusted for age (as a linear term), sex and income. We ran separate sets of models for each modifier of interest: self-reported physical limitations, age and SF12-P score. We checked for a three-way interaction between SBP, physical limitations and age $(<65 \mathrm{vs}\rangle=65)$ in race-specific models, but did not find any evidence that the association between SBP and physical limitations on incident CVD varied by age category.

To assess modification on a multiplicative scale, we ran Poisson models stratified by functional status (eg, with physical limitations; without physical limitations; age $<65$; age $\geq 65$ ) and by race/ethnicity to estimate the association between SBP (as a continuous measure) and CVD. We tested for interaction by running models with an interaction term between SBP and the measure of functional status. We explored the impact of adjusting for additional covariates (smoking, diabetes, antihypertensive medication, statins, total cholesterol, DBP and BMI) among white and black subgroups only, due to limited sample size. Due to conceptual concern over distinguishing confounders from mediators in the joint effects of SBP and functional status on CVD, we present the minimally adjusted models as our primary results.

We also explored the relationship between SBP and CVD by functional status on an additive scale, because departures from multiplicativity may result from different baseline levels of risk in the subgroups of interest. We ran Poisson models with SBP categorised into quintiles, in order to estimate the incidence rate of CVD at each quintile. Models of physical limitation were stratified (with limitations vs without limitations) and adjusted for age. In the models for age, incidence rates were estimated at the first and third quartile (ages 54 and 70, respectively). We used multivariable regression spline models to evaluate the best shape (linear or non-linear) of the trend in incidence rate across quintiles. We calculated slopes across quintiles to represent the average incidence rate difference (IRD) per quintile. All analyses were done in Stata V.13.1.

\section{RESULTS}

Our analysis excluded 584 participants who did not have a blood pressure measurement at examination 2, 14 participants who did not have physical function measures at examination 2, 5 participants with missing information on incident CVD and 3 participants who had prevalent CVD at examination 2. Excluded participants were older (mean age 67.9 vs 63.5$)$, more likely to be male $(61.2 \%$ vs $47.4 \%$ ), less well educated ( $22.7 \%$ vs $36.7 \%$ with bachelor's or higher), had lower incomes $(2.0 \%$ vs $22.6 \%$ with income $>$ US $\$ 75000$ ) and were more likely to report physical limitations $(50.5 \%$ vs $27.3 \%)$. SBP, DBP and racial/ethnic distribution were similar among excluded and included participants. Among the analytic sample of 6117 participants, age at baseline ranged from 46 to 87. Participants were followed for an average of 8.8 years (until time of CVD incidence, death, loss to follow-up or 31 December 2012), during which time there were 557 incident cases of CVD detected.

White participants were older, more educated and had higher income levels than members of other racial/ethnic groups (table 1). Black participants had the highest mean SBP and were most likely to be on antihypertensive medications. Black participants were most likely to report physical limitations, while white participants were most likely to be diagnosed with CVD during follow-up.

We observed a generally weaker association between SBP and CVD among low-functioning subgroups (those with physical limitations or over age 65) compared with high-functioning subgroups (table 2). This pattern was most apparent among white participants, though there was a similar pattern among black participants that did not reach statistical significance for the interaction of SBP and physical limitations. Among Hispanic and Chinese participants, the association between SBP and CVD appeared not to be modified by measures of functional status. Among all racial/ethnic groups, higher SBP was associated with higher incidence of CVD. There was no evidence for an interaction between SBP, age and physical limitation among white $(\mathrm{P}=0.71)$ or black $(\mathrm{P}=0.22)$ participants. Sample sizes were insufficient to estimate the three-way interaction among Hispanic or Chinese participants.

The estimated CVD incidence rates by quintile of SBP revealed more nuances about the race/ethnicity-specific associations between SBP, functional status and CVD (figure 1). Among all racial/ethnic groups, the incidence of CVD was higher among low-functioning subgroups (with physical limitations or older age) than high-functioning subgroups (without physical limitations or younger age). Multivariable spline regression models reported that a linear fit was appropriate for the association between SBP and CVD in all subgroups.

Among white participants, the estimated increase in CVD per quintile higher SBP was smaller for those with physical limitations than for those without physical limitations (IRD 1.67 vs 3.35 per 1000 person-years). However, the average IRD across quintiles of SBP was slightly larger 
Table 1 Characteristics of analytic sample at Multi-Ethnic Study of Atherosclerosis examination 2 by race/ethnicity

\begin{tabular}{|c|c|c|c|c|c|}
\hline & White & Black & Hispanic & Chinese & $P$ value \\
\hline $\mathrm{N}$ & 2421 & 1659 & 1320 & 717 & \\
\hline Age (years) & 64.1 & 63.5 & 62.7 & 63.3 & $<0.001$ \\
\hline Female & $51.6 \%$ & $54.9 \%$ & $52.3 \%$ & $51.3 \%$ & 0.17 \\
\hline \multicolumn{6}{|l|}{ Income } \\
\hline$<U S \$ 16000$ & $10.0 \%$ & $19.9 \%$ & $38.6 \%$ & $44.5 \%$ & \\
\hline US\$16 000-US\$39999 & $21.2 \%$ & $32.0 \%$ & $35.3 \%$ & $22.4 \%$ & \\
\hline US\$40 000-US\$74999 & $30.9 \%$ & $30.1 \%$ & $18.6 \%$ & $15.4 \%$ & \\
\hline US\$75 000+ & $37.8 \%$ & $18.0 \%$ & $7.5 \%$ & $17.7 \%$ & $<0.001$ \\
\hline \multicolumn{6}{|l|}{ Education } \\
\hline High school or less & $21.1 \%$ & $29.9 \%$ & $63.7 \%$ & $39.1 \%$ & \\
\hline Some college or Associate's & $27.8 \%$ & $35.2 \%$ & $26.0 \%$ & $20.3 \%$ & \\
\hline Bachelor's or more & $51.1 \%$ & $34.9 \%$ & $10.3 \%$ & $40.6 \%$ & $<0.001$ \\
\hline BMI $\left(\mathrm{kg} / \mathrm{m}^{2}\right)$ & 27.7 & 30.1 & 29.6 & 24.1 & $<0.001$ \\
\hline Current smoker & $10.9 \%$ & $16.0 \%$ & $10.2 \%$ & $5.6 \%$ & $<0.001$ \\
\hline Total cholesterol (mg/dL) & 192.8 & 188.4 & 194.1 & 189.9 & $<0.001$ \\
\hline Taking statins & $23.5 \%$ & $18.8 \%$ & $16.6 \%$ & $17.2 \%$ & $<0.001$ \\
\hline \multicolumn{6}{|l|}{ Diabetes } \\
\hline Impaired fasting glucose & $17.9 \%$ & $20.1 \%$ & $21.7 \%$ & $20.6 \%$ & \\
\hline Treated diabetes & $5.9 \%$ & $16.6 \%$ & $16.6 \%$ & $11.2 \%$ & $<0.001$ \\
\hline DBP (mm Hg) & 68.9 & 73.5 & 70.1 & 69.3 & $<0.001$ \\
\hline Taking antihypertension medication & $37.8 \%$ & $54.0 \%$ & $37.3 \%$ & $32.1 \%$ & $<0.001$ \\
\hline SBP, mean & 121.0 & 130.2 & 125.0 & 120.7 & $<0.001$ \\
\hline \multicolumn{6}{|l|}{ SBP quintiles } \\
\hline Quintile $1(60-107 \mathrm{~mm} \mathrm{Hg})$ & $24.3 \%$ & $12.2 \%$ & $20.7 \%$ & $27.8 \%$ & \\
\hline Quintile $2(107.5-116.5 \mathrm{~mm} \mathrm{Hg})$ & $23.3 \%$ & $16.8 \%$ & $20.2 \%$ & $19.4 \%$ & \\
\hline Quintile $3(117-127 \mathrm{~mm} \mathrm{Hg})$ & $19.2 \%$ & $19.8 \%$ & $18.7 \%$ & $18.0 \%$ & \\
\hline Quintile 4 (127.5-141 mm Hg) & $19.0 \%$ & $23.7 \%$ & $20.0 \%$ & $18.6 \%$ & \\
\hline Quintile 5 (141.5-230 mm Hg) & $14.2 \%$ & $27.6 \%$ & $20.5 \%$ & $16.3 \%$ & $<0.001$ \\
\hline Self-reported physical limitations & $26.4 \%$ & $31.7 \%$ & $27.2 \%$ & $20.8 \%$ & $<0.001$ \\
\hline Accomplished less than liked & $23.1 \%$ & $27.8 \%$ & $24.6 \%$ & $19.1 \%$ & \\
\hline Limited in work or daily activities & $17.2 \%$ & $20.7 \%$ & $21.8 \%$ & $16.5 \%$ & \\
\hline SF12-P score & 49.9 & 47.8 & 47.9 & 48.9 & $<0.001$ \\
\hline Incident CVD & $10.2 \%$ & $8.9 \%$ & $9.2 \%$ & $5.7 \%$ & $<0.01$ \\
\hline $\begin{array}{l}\text { Mean time to CVD or end of follow-up } \\
\text { (years) }\end{array}$ & 8.9 & 8.6 & 8.7 & 9.0 & $<0.001$ \\
\hline
\end{tabular}

BMI, body mass index; CVD, cardiovascular disease; DBP, diastolic blood pressure; SBP, systolic blood pressure; SF12-P, Physical Component Summary score.

among older whites (estimated at age 70) compared with younger whites (estimated at age 54). Among black participants, the association of SBP and CVD was weaker both among participants with physical limitations and among older participants, compared with those without physical limitations and younger participants. In contrast, among Hispanic participants, those with physical limitations and those of older age had larger IRDs (a larger increase in CVD incidence per quintile higher SBP) than those with no physical limitations and at younger ages, respectively.
Large CIs limit generalisations among Chinese participants. Chinese participants were less likely to report physical limitations and less likely to develop CVD than other racial/ethnic groups, further hindering statistical power.

Adjustment for additional covariates resulted in generally attenuated but consistent results among white and black participants (figure 2). The adjusted association of SBP with CVD risk was statistically significant among those with high functional status, but approached unity in those with physical limitations or age $\geq 65$. 
Table 2 Estimated associations (incident rate ratio (IRR)* per $10 \mathrm{~mm} \mathrm{Hg}$ higher systolic blood pressure (SBP) between SBP and incident cardiovascular disease (CVD) by measures of functional status and racial/ethnic group

\begin{tabular}{|c|c|c|c|c|}
\hline & $\begin{array}{l}\text { No physical } \\
\text { limitations }\end{array}$ & With physical limitations & Age $<65$ & Age $\geq 65$ \\
\hline \multicolumn{5}{|l|}{ Overall } \\
\hline $\mathrm{N}$ & 4446 & 1671 & 3218 & 2899 \\
\hline IRR & 1.21 & 1.10 & 1.30 & 1.11 \\
\hline $95 \% \mathrm{Cl}$ & 1.16 to 1.27 & 1.03 to 1.17 & 1.22 to 1.39 & 1.06 to 1.17 \\
\hline$P$ value for interaction & 0.04 & & $<0.01$ & \\
\hline \multicolumn{5}{|l|}{ White } \\
\hline $\mathrm{N}$ & 1783 & 638 & 1229 & 1192 \\
\hline IRR & 1.29 & 1.09 & 1.33 & 1.17 \\
\hline $95 \% \mathrm{Cl}$ & 1.19 to 1.40 & 0.99 to 1.20 & 1.18 to 1.49 & 1.01 to 1.07 \\
\hline$P$ value for interaction & $<0.01$ & & $<0.01$ & \\
\hline \multicolumn{5}{|l|}{ Black } \\
\hline $\mathrm{N}$ & 1134 & 525 & 864 & 795 \\
\hline IRR & 1.25 & 1.10 & 1.36 & 1.10 \\
\hline $95 \% \mathrm{Cl}$ & 1.14 to 1.37 & 0.96 to 1.25 & 1.22 to 1.52 & 1.00 to 1.21 \\
\hline$P$ value for interaction & 0.14 & & $<0.01$ & \\
\hline \multicolumn{5}{|l|}{ Hispanic } \\
\hline $\mathrm{N}$ & 961 & 359 & 740 & 580 \\
\hline IRR & 1.11 & 1.10 & 1.13 & 1.08 \\
\hline $95 \% \mathrm{Cl}$ & 1.00 to 1.23 & 0.95 to 1.27 & 0.97 to 1.31 & 0.98 to 1.19 \\
\hline$P$ value for interaction & 0.89 & & 0.66 & \\
\hline \multicolumn{5}{|l|}{ Chinese } \\
\hline $\mathrm{N}$ & 568 & 149 & 385 & 332 \\
\hline IRR & 1.21 & 1.23 & $-\dagger$ & 1.10 \\
\hline $95 \% \mathrm{Cl}$ & 1.01 to 1.44 & 0.95 to 1.58 & $-\dagger$ & 0.94 to 1.29 \\
\hline$P$ value for interaction & 0.93 & & - & \\
\hline
\end{tabular}

*From Poisson models with offset for person-time contributed until incident CVD, death or loss to follow-up. Models for physical limitations were adjusted for age (continuous), gender and income (dichotomised at US $\$ 75000$. Models by age category were adjusted for gender and income (dichotomised at US $\$ 75,000$ ).

†Omitted due to small number of events $(<10)$.

Using SF12-P scores to measure functional status produced similar patterns to those in figure 1 but with smaller differences between low-functioning (estimated at $\mathrm{SF} 12-\mathrm{P}=44.5)$ and high-functioning $(\mathrm{SF} 12-\mathrm{P}=55.5)$ groups (see online supplementary appendix figure 1 ).

\section{DISCUSSION}

Overall, we observed attenuated associations between SBP and incident CVD among those with low functional status (measured by self-reported physical limitations and by chronological age) among white and black adults. This pattern was generally consistent across both multiplicative and additive scales using self-reported physical limitations, but not age (as older white participants had a slightly larger increase in risk per quintile higher SBP than younger white participants). Our findings are consistent with previous research that has found attenuated or inverted associations between blood pressure and health outcomes among low-functioning subgroups (defined by age, walking speed or limitations in activities of daily living). ${ }^{3418}$

Blood pressure treatment guidelines and randomised controlled trials have frequently used age to define treatment targets or populations of interest. ${ }^{19-22}$ Chronological age is an imprecise measure of functional status, though it is easily and routinely collected. Others have recognised the limitations of relying on chronological age to predict health-related outcomes. ${ }^{23}$ Among white participants, using self-reported physical limitations as a measure of functional status provided consistent evidence of attenuated associations between SBP and incident CVD among adults with physical limitations across multiplicative and additive scales, while there was not an attenuated association between SBP and incident CVD among older 
Figure 1 Estimated incidence rates of cardiovascular disease by quintile of systolic blood pressure, by measures of functional status and race/ethnicity. PY, per 1000 person years.

adults on an additive scale. Among blacks, both older age and physical limitations revealed attenuated associations between SBP and CVD on both multiplicative and additive scales (though not statistically significant for physical limitations on a multiplicative scale). Additionally, across all race/ethnic groups, those with physical limitations had higher incidence of CVD at all levels of SBP than those without physical limitations, after adjusting for age. Where feasible, self-reported physical limitations or other specific measures of functional status may be a useful addition to methods of assessing risk in clinical settings.

We observed no apparent patterning of SBP and CVD by functional status among Hispanics or Chinese participants. For Chinese participants, the small sample size is likely a key limitation for distinguishing patterns by functional status. Additionally, Chinese participants were least likely to report physical limitations and had the lowest incidence of CVD. For Hispanic participants, associations 


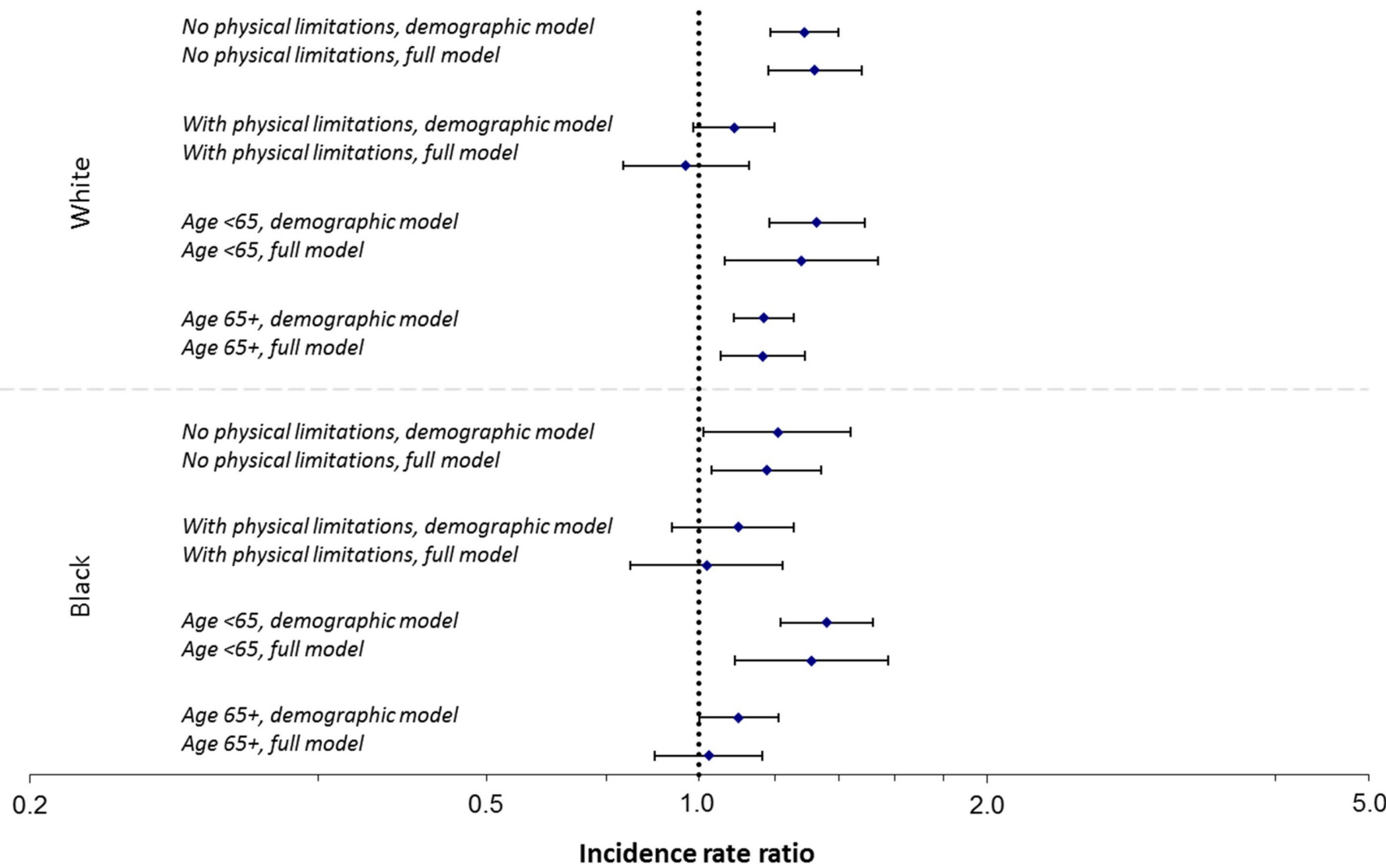

Higher SBP protective

Higher SBP harmful

Figure 2 Forest plot of adjusted incident rate ratio for incident cardiovascular disease per $10 \mathrm{~mm} \mathrm{Hg} \mathrm{higher} \mathrm{systolic} \mathrm{blood}$ pressure (SBP) among white and black participants.

between SBP and CVD were similar by functional status on a multiplicative scale, and on an additive scale appeared to be stronger (larger average IRDs across quintiles of SBP) for those with physical limitations and those at higher ages than those without physical limitations and at lower ages, respectively. Hispanic participants were also the youngest of the racial/ethnic groups in MESA. Previous research in a Hispanic population found that the association between SBP and all-cause mortality was attenuated among slow walkers compared with fast walkers; however, these participants were older (mean age 70.5 years) and less healthy overall (mean SBP $139 \mathrm{~mm} \mathrm{Hg}, 22.8 \%$ on diabetes medication) than MESA's Hispanic participants (mean age 62.7 years, mean SBP $125 \mathrm{~mm} \mathrm{Hg}, 16.3 \%$ on diabetes medication). Future research should continue to investigate patterns of SBP and CVD risk among diverse minority populations.

Some limitations are important to consider. Self-reported physical limitations, assessed by two questions, is a crude measure of functional status; more objective measures may demonstrate even better discrimination in CVD risk. Additionally, previous research has shown that the health risks of high SBP among those with functional impairments may be stronger among older adults than among middle-aged adults. ${ }^{4}$ We did not find any evidence that the association between SBP and physical limitations varied by age $(<65$ vs $\geq 65)$, though we may have been underpowered to detect heterogeneity statistically.

Additionally, more research is needed to inform how observed differences in CVD risk associated with SBP should influence treatment of high blood pressure. The biological mechanism mediating an attenuated association between high blood pressure and outcomes among low-functioning older adults remains uncertain, although there are several plausible explanations. Poor physical functioning may be associated with compromised haemodynamic regulation, vascular stiffening and insufficient cerebral, myocardial or renal perfusion, resulting in poorer health outcomes independent of SBP. Others have noted the challenges in accurate measurement of blood pressure in older adults, due to orthostatic hypotension, pseudohypertension and postprandial hypertension, ${ }^{24}$ suggesting that attenuated association with outcomes may reflect measurement error. Another possibility is that treatment of high blood pressure, which often requires multiple medications, could result in polypharmacy or other adverse events such as falls and fractures, ${ }^{25}$ and these adverse events could initiate a cascade of events that could result in hospitalisation, morbidity and even death. Thus, observed low blood pressures may be correlated poorer outcomes. Our analysis shows that future studies that evaluate benefits of treating high blood pressure 
should explore patterns by race/ethnicity as well as functional status.

The presence of racial/ethnic variation in associations between SBP, incident CVD and functional status do not imply biologically predetermined differences in risks; rather, our findings should caution against generalising results from predominantly white study populations to other racial/ethnic populations. The observed differences that we found are likely the result of broad social and environmental influences throughout the life course that are strongly patterned by racial/ethnic identity in the USA. However, these findings are also novel and, if replicated, much more research is needed to understand the causal pathways resulting in racial/ethnic heterogeneity in associations between SBP, functional status and CVD.

In summary, we found that the risk of incident CVD associated with high blood pressure appears to be attenuated among white and black adults with physical limitations and at older ages in a diverse cohort of middle aged and older adults. Patterns among Hispanic and Chinese adults were less clear, which likely reflects limited sample sizes. Understanding how functional status (or factors underlying functional impairments) influence CVD risk across racial/ethnic groups could be useful for identifying at-risk persons and informing public health strategies to improve health and reduce CVD risk.

\section{Author affiliations}

${ }^{1}$ School of Biological and Population Health Sciences, Oregon State University, Corvallis, Oregon, USA

${ }^{2}$ Department of Medicine, Epidemiology and Biostatistics, University of California, San Francisco, California, USA

${ }^{3}$ Department of Biostatistics, University of Washington, Seattle, Washington, USA ${ }^{4}$ General Internal Medicine Section, Veterans Affairs Medical Center, San Francisco, California, USA

${ }^{5}$ Cardiovascular Health Research Unit, Departments of Medicine, Epidemiology, andHealth Services, University of Washington and Kaiser Permanente Washington Health Research Institute, Seattle, Washington, USA

Acknowledgements The authors thank the other investigators, the staff and the participants of the MESA study for their valuable contributions. A full list of participating MESA investigators and institutions can be found at http://www.mesanhlbi.org.

Contributors PK developed the initial research question, conducted all analyses and drafted the manuscript. MCO provided oversight, including review of analyses and editing the manuscript. CP, RK, MS and BMP reviewed the results and provided guidance on interpretation, and provided comments on multiple drafts of the manuscript.

Funding This research was supported by grants K01AG039387 and R01AG46206 from the National Institute on Aging; contracts HHSN268201500003I, N01-HC-95159, N01-HC-95160, N01-HC- 95161, N01-HC-95162, N01-HC-95163, N01-HC-95164, N01-HC-95165, N01-HC95166, N01- HC-95167, N01-HC-95168 and N01-HC-95169 from the National Heart, Lung, and Blood Institute, and grants UL1-TR-000040, UL1-TR-001079 and UL1-TR-001420 from NCATS.

Competing interests None declared.

Patient consent Obtained.

Ethics approval IRBs at each participating site.

Provenance and peer review Not commissioned; externally peer reviewed.

Data sharing statement No additional unpublished data are available. More information about MESA, including all participating MESA investigators and institutions, can be found at http://www.mesa-nhlbi.org.
Open Access This is an Open Access article distributed in accordance with the Creative Commons Attribution Non Commercial (CC BY-NC 4.0) license, which permits others to distribute, remix, adapt, build upon this work non-commercially, and license their derivative works on different terms, provided the original work is properly cited and the use is non-commercial. See: http://creativecommons.org/ licenses/by-nc/4.0/

C Article author(s) (or their employer(s) unless otherwise stated in the text of the article) 2018. All rights reserved. No commercial use is permitted unless otherwise expressly granted.

\section{REFERENCES}

1. Mozaffarian D, Benjamin EJ, Go AS, et al. Heart disease and stroke statistics-2016 update: a report from the american heart association. Circulation 2016;133:CIR. 0000000000000350.

2. Odden MC, Covinsky KE, Neuhaus JM, et al. The association of blood pressure and mortality differs by self-reported walking speed in older Latinos. J Gerontol A Biol Sci Med Sci 2012;67:977-83.

3. Odden MC, Peralta CA, Haan MN, et al. Rethinking the association of high blood pressure with mortality in elderly adults: the impact of frailty. Arch Intern Med 2012;172:1162-8.

4. Windham BG, Griswold ME, Lirette S, et al. Effects of age and functional status on the relationship of systolic blood pressure with mortality in mid and late life: the ARIC study. J Gerontol A Biol Sci Med Sci 2017;72:89-94.

5. Peralta CA, Katz R, Newman AB, et al. Systolic and diastolic blood pressure, incident cardiovascular events, and death in elderly persons: the role of functional limitation in the cardiovascular health study. Hypertension 2014;64:472-80.

6. Sabayan B, van Vliet P, de Ruijter W, et al. High blood pressure, physical and cognitive function, and risk of stroke in the oldest old: the leiden 85-plus study. Stroke 2013;44:15-20.

7. Geronimus AT, Hicken M, Keene D, et al. "Weathering" and age patterns of allostatic load scores among blacks and whites in the United States. Am J Public Health 2006;96:826-33.

8. Howard G, Lackland DT, Kleindorfer DO, et al. Racial differences in the impact of elevated systolic blood pressure on stroke risk. JAMA Intern Med 2013;173:46-51.

9. Christine PJ, Auchincloss AH, Bertoni AG, et al. Longitudinal associations between neighborhood physical and social environments and incident type 2 diabetes mellitus: the MultiEthnic Study of Atherosclerosis (MESA). JAMA Intern Med 2015;175:1311-20.

10. Bild DE, Bluemke DA, Burke GL, et al. Multi-ethnic study of atherosclerosis: objectives and design. Am J Epidemiol 2002;156:871-81.

11. Perloff D, Grim C, Flack J, et al. Human blood pressure determination by sphygmomanometry. Circulation 1993;88:2460-70.

12. Ware J, Kosinski M, Keller SD. A 12-item short-form health survey: construction of scales and preliminary tests of reliability and validity. Med Care 1996;34:220-33.

13. Jenkinson $C$, Layte $R$, Jenkinson $D$, et al. A shorter form health survey: can the SF-12 replicate results from the SF-36 in longitudinal studies? J Public Health Med 1997;19:179-86.

14. Gandek B, Ware JE, Aaronson NK, et al. Cross-validation of item selection and scoring for the SF-12 Health Survey in nine countries: results from the IQOLA Project. International quality of life assessment. J Clin Epidemiol 1998;51:1171-8.

15. Ware JE, Kosinski M, Turner-Bowker DM, et al. How to score version 2 of the SF-12 health survey (with a supplement documenting version 1): Quality Metric Incorporated, 2002.

16. Folsom AR, Kronmal RA, Detrano RC, et al. Coronary artery calcification compared with carotid intima-media thickness in the prediction of cardiovascular disease incidence: the Multi-Ethnic Study of Atherosclerosis (MESA). Arch Intern Med 2008;168:1333-9.

17. Yeboah J, Folsom AR, Burke GL, et al. Predictive value of brachial flow-mediated dilation for incident cardiovascular events in a population-based study: the multi-ethnic study of atherosclerosis. Circulation 2009;120:502-9.

18. Peralta CA, Katz R, Newman AB, et al. Systolic and diastolic blood pressure, incident cardiovascular events, and death in elderly persons: the role of functional limitation in the Cardiovascular Health Study. Hypertension 2014;64:472-80.

19. James PA, Oparil S, Carter BL, et al. 2014 evidence-based guideline for the management of high blood pressure in adults: report from the panel members appointed to the Eighth Joint National Committee (JNC 8). JAMA 2014;311:507-20. 
20. Wright JT, Williamson JD, Whelton PK, et al. A randomized trial of intensive versus standard blood-pressure control. N Engl J Med 2015;373:2103-16.

21. Beckett NS, Peters R, Fletcher AE, et al. Treatment of hypertension in patients 80 years of age or older. N Engl J Med 2008;358:1887-98.

22. Turnbull F, Neal B, Ninomiya T, et al. Effects of different regimens to lower blood pressure on major cardiovascular events in older and younger adults: meta-analysis of randomised trials. BMJ 2008;336:1121-3.
23. Levine ME. Modeling the rate of senescence: can estimated biological age predict mortality more accurately than chronological age? J Gerontol A Biol Sci Med Sci 2013;68:667-74.

24. Morley JE. Systolic hypertension should not be treated in persons aged 80 and older until blood pressure is greater than $160 \mathrm{mmHg}$. $J$ Am Geriatr Soc 2013;61:1197-8.

25. Tinetti ME, Han L, Lee DS, et al. Antihypertensive medications and serious fall injuries in a nationally representative sample of older adults. JAMA Intern Med 2014;174:588-95. 